\title{
Sadness and mild cognitive impairment as predictors for interferon-alpha-induced depression in patients with hepatitis C
}

\author{
Susanne Sarkar, Rahul Sarkar, Thomas Berg and Martin Schaefer
}

\section{Background}

Antiviral therapy with interferon-alpha (IFN- $\alpha$ ) for hepatitis C virus $(\mathrm{HCV})$ infection is associated with increased risk for depression.

\section{Aims \\ To identify clinical predictors for IFN- $\alpha$-induced depression during antiviral therapy for HCV infection.}

\section{Method}

Depression (defined with the Montgomery-Åsberg Depression Rating Scale (MADRS)) was evaluated before and during antiviral treatment in 91 people with chronic HCV infection without a history of psychiatric disorders. Cognitive function was evaluated using the Trail Making Test A/B (TMT A/B). (Trial registration at ClinicalTrials.gov: NCT00136318.)

\section{Results}

Depression during antiviral therapy was significantly associated with a baseline MADRS score of 3 or higher $(P=0.006)$. In total, $89 \%(n=16)$ of patients who had a baseline score $>0$ for the single item sadness developed depression. Poor baseline performance in the TMT A $(P=0.027)$ and TMT B $(P=0.033)$ was predictive for severe depression.

\section{Conclusions}

Pre-treatment screening for subthreshold depressive and cognitive symptoms will help to identify those at risk for IFN- $\alpha$-associated depression among patients with chronic hepatitis $\mathrm{C}$.

\section{Declaration of interest}

M.S.: consultancy and member of speakers' bureau at Roche Pharma (Germany/Switzerland), Janssen-Cilag, Servier and Shire. T.B.: consultancy or advisory board member, speakers' bureau at Abbott, Bristol-Myers Squibb, Boehringer Ingelheim, Gilead Sciences, Janssen Pharmaceuticals, Merck \& Co, Novartis, Roche and Vertex Pharmaceuticals Incorporated; research grants from Gilead Sciences, Janssen Pharmaceuticals, Novartis and Roche.
The combination of pegylated (PEG) interferon-alpha (IFN- $\alpha$ ) plus ribavirin is established standard treatment of hepatitis $\mathrm{C}$ virus (HCV) infection. Antiviral therapy including IFN- $\alpha$, however, is associated with significant psychiatric side-effects such as depressive symptoms, fatigue and insomnia. ${ }^{1-3}$ The reported prevalence of INF-associated major depressive episodes is $20-40 \%$, and up to $70 \%$ of patients may develop mild to moderate depressive episodes with a significant impact on their quality of life. This is likely to negatively affect adherence to treatment and may represent a major cause of treatment discontinuation. ${ }^{3,4}$ Recently, pre-emptive treatment with antidepressants, notably selective serotonin reuptake inhibitors (SSRIs), demonstrated a reduction of the incidence of mild to severe major depressive episodes in HCV-infected patients during antiviral treatment. ${ }^{5}$ The largest trial in patients without prior psychiatric disorders to date was conducted with escitalopram. When compared with controls without pre-emptive treatment, a lower incidence and severity of depression, defined by the presence of DSM-IV major depressive episodes criteria and Montgomery-Åsberg Depression Rating Scale (MADRS) scores, was observed during HCV treatment. ${ }^{6}$ Although antidepressant pre-treatment showed a high efficacy in reducing overall depression rates and the use of SSRIs was safe without negative effects on antiviral response, it also needs to be considered that a significant proportion of patients do not develop clinically relevant depressive episodes. Thus, a pharmacological prophylactic treatment might put them at risk of harm without apparent benefits. Concomitant treatment with antidepressants may cause additional side-effects including sexual dysfunction, insomnia, nausea and visual and cardiac symptoms. ${ }^{7}$ Hence, pre-emptive antidepressant treatment should be limited to patients who are at increased risk for depression, and reliable identification of high-risk patients for INF-associated depressive syndromes is warranted. Screening criteria to identify those patients who might have the greatest benefits from pre-emptive antidepressant therapy are warranted for limiting potential disadvantages and maximising potential benefits of psychopharmacological pre-treatment in routine care. The aim of the present post hoc analysis was to identify neuropsychiatric risk factors that predict the development of depression during antiviral treatment with IFN- $\alpha$.

\section{Method}

\section{Settings and participants}

This article reports on a total of 91 people with chronic HCV infection who participated in a prospective controlled trial investigating the prevention of depressive symptoms with escitalopram during antiviral treatment with PEG-IFN- $\alpha$ and ribavirin (for detailed information see Schaefer et $a l^{6}$ ) and who were randomised to placebo treatment.

All patients were treatment-naive to $\mathrm{HCV}$ treatment, older than 18 years and had a chronic HCV infection with serum HCV-RNA levels of $1000 \mathrm{IU} / \mathrm{mL}$ or higher. Psychiatric exclusion criteria were a lifetime diagnosis of an affective disorder, drug misuse in the past 12 months, treatment with antidepressants during the past 3 years or a history of any other Axis I disorder according to DSM-IV criteria. ${ }^{8}$ Medical exclusion criteria were pre-treatment with IFN or immunotherapy, other chronic infections or a severe somatic comorbid condition. The study protocol was approved by the ethics committee of the Charite University Medicine Berlin and confirmed by local ethics 
committees. The study was conducted in accordance with the principles of the Declaration of Helsinki and local laws and regulations. All participants provided written informed consent. The trial is registered at ClinicalTrials.gov: NCT00136318.

\section{Treatment}

Eligible patients participated in a multicentre, double-blind, prospective, randomised and placebo-controlled phase-III study consisting of three different study periods as described elsewhere. ${ }^{6}$ The original study compared pre-emptive treatment with escitalopram $v$. placebo in patients undergoing antiviral therapy with PEG-IFN- $\alpha 2$ a plus ribavirin; here we report on the 91 patients who had been randomised to placebo. During a pretreatment period of 14 weeks before antiviral therapy was started, patients were monitored for spontaneously developing symptoms of depression. Next, all patients received antiviral therapy with PEG-IFN- $\alpha 2$ a plus ribavirin (Roche, Grenzach-Wyhlen, Germany). Patients with HCV genotype 1 or 4 were treated for 48 weeks with PEG-IFN- $\alpha 2 \mathrm{a}$ at a dose of $180 \mu \mathrm{g}$ per week, and ribavirin at a dose of $1000 \mathrm{mg}$ per day (body weight $<75 \mathrm{~kg}$ ) or $1200 \mathrm{mg}$ per day (body weight $\geqslant 75 \mathrm{~kg}$ ). Patients with $\mathrm{HCV}$ genotype 2 or 3 received PEG-IFN- $\alpha 2 \mathrm{a}(180 \mu \mathrm{g} /$ week $)$ and ribavirin $(800 \mathrm{mg}$ daily) for 24 weeks. Doses of ribavirin and PEG-IFN- $\alpha 2$ a could be adjusted in response to side-effects. Benzodiazepines were not allowed during the trial.

\section{Psychiatric assessments and end-points}

Psychiatric assessments were performed 14, 8, and 2 weeks before antiviral therapy was started as well as after 2, 4, 12 and 24 and, for patients with genotypes 1 and 4, 48 weeks of antiviral therapy and, finally, 24 weeks after antiviral treatment (follow-up assessment). The Mini-International Neuropsychiatric Interview was used at baseline as a screening tool to detect pre-existing psychiatric disorders. $^{9}$ The Structured Clinical Interview for DSM-IV Disorders was used to verify the incidence of major depressive disorder before and during treatment. ${ }^{10}$ In addition we used the MADRS $^{11}$ and the 21-item Beck Depression Inventory (BDI) $)^{12}$ to define and identify the incidence of all clinically relevant (mild to moderate) depressive symptoms. The MADRS scale consists of 10 items assessing symptoms associated with depression (apparent and reported sadness, inner tension, reduced sleep, reduced appetite, loss of concentration, lassitude, inability to feel, pessimistic thoughts and suicidal thoughts), each item scored from 0 to 6 according to severity. We applied three different definitions of depressive mood disorder: (a) those who fulfilled criteria of a current major depressive episode according to DSM-IV (independent of severity), and two groups defined on the basis of MADRS scores independent of fulfilling full DSM-IV major depressive episodes criteria; (b) MADRS scores $\geqslant 13$ were used as cut-off for clinically relevant depression; (c) MADRS $\geqslant 25$ for severe depression. ${ }^{6,11,13}$ In addition, a self-rating instrument, the BDI was administered to screen for obvious differences in clinician-rated MADRS scores and patient's account and to identify single items associated with the later development of depression under treatment. The Trail Making Test A/B (TMT $\mathrm{A} / \mathrm{B}$ ) and the Regensburger verbal fluency test were applied to evaluate cognitive function at baseline. ${ }^{14,15}$ In addition, language production was assessed using two different word fluency tasks of the Regensburger verbal fluency test, measuring semantic and phonematic word fluency. The Clinical Assessment Geriatric Scale (also called Sandoz Clinical Assessment-Geriatric (SCAG)) was applied to measure mental-mnestic disturbances (confusion, mental alertness, impairment of recent memory, disorientation) with higher values indicating greater impairment. ${ }^{16}$

\section{Statistical analysis}

Descriptive analysis was applied for the frequencies of primary outcome parameters (major depressive episodes, MADRS score $\geqslant 13, \geqslant 25$, sustained virologic response and adverse events). Chi-squared tests were used for categorical data and $t$-test for continuous data to compare the rate of patients with and without depressive symptomatology (according to MADRS score $\geqslant 13$, $\geqslant 25$ and DSM-IV criteria), baseline characteristics (age, gender, body mass index (BMI), genotype 1 and 4 v. 2 and 3, baseline MADRS score, BDI score, TMT A/B, word fluency, SCAG, study site (four major sites; all other small sites were combined into one category)) and MADRS score at week 2 and 4 after the beginning of IFN therapy. Multiple logistic regression analysis was used to calculate odds ratios of the incidence of IFN-associated depressive symptomatology (MADRS $\geqslant 13$, major depressive episodes, MADRS $\geqslant 25$ ) adjusted for gender, age, BMI, genotype ( 1 and 4 v. 2 and 3), baseline MADRS $>3$ and baseline BDI $>4$. We further evaluated single-item scores of the MADRS scale to screen for potential risk factors for developing depressive episodes during antiviral therapy. To evaluate the relationship between specific subclinical depressive symptoms at baseline and the development of an IFN-associated depressive syndrome (MADRS scores $\geqslant 13$, major depressive episodes, MADRS scores $\geqslant 25$ ), items measured on the MADRS scale were used to perform different regression trees. Independent variables were the 10 single items of the MADRS scale (apparent and reported sadness, inner tension, inability to feel, loss of concentration, pessimistic thoughts, reduced sleep, reduced appetite, lassitude and suicidal thoughts). On the basis of decision trees, we defined cut-off points for each symptom of depression of the MADRS scale at baseline to discriminate between patients with or without IFN-associated depression. There is a need for cut-off points for subclinical depressive symptoms to construct rules for making predictions about individual cases and assist in identifying homogeneous groups with high or low risk for the development of IFNassociated depression. To evaluate the influence of subclinical cognitive dysfunction at baseline, multiple logistic regression analysis was used to calculate the odds ratios of the incidence of IFN-associated depression (MADRS $\geqslant 13$, major depressive episodes, MADRS $\geqslant 25$ ) adjusted for TMT A/B, semantic and phonematic word fluency and the SCAG score.

For the primary end-point (MADRS score $\geqslant 13$ ), we dealt with missing MADRS assessments by multiple imputation. We generated a mean of 10 imputed samples by a linear model using all MADRS assessments, age and BMI. For each patient and sample, the maximum MADRS score was subsequently computed and dichotomised at a MADRS score of 13. For all imputed samples, a logistic model tested differences between treatment groups with the sole factor group. Combined point estimates and the covariance matrix of the model parameters were subsequently calculated by the Rubin rule. Finally, the combined $P$-value, difference of depression rates with $95 \%$ confidence intervals were calculated by the delta method. The multiple imputation samples were generated by using SPSS version 19.01 for Windows. Patients without HCV RNA measurements at follow-up were considered non-responders to antiviral treatment. All tests and confidence intervals were twosided, and the level of significance was $\alpha=0.05$. A statistical analysis was performed by using SPSS Statistics, version 19.0 for Windows.

\section{Results}

\section{Development of depressive symptoms during antiviral treatment}

None of the 91 patients exhibited any clinically relevant depressive syndromes (defined as a MADRS score $\geqslant 13$ ) before the initiation 
of INF treatment. A total of $88 \%$ (80/91) of the patients finished antiviral therapy according to the protocol. The overall incidence of adverse events was $87 \%$. Dose reduction of PEG-IFN- $\alpha 2$ a was necessary for $19 \%(n=17)$ of patients and ribavirin dose was decreased in $29 \%(n=26)$ of patients. Of the 91 patients, $54 \%$ ( $n=49,95 \%$ CI 48-69\%) developed MADRS scores $\geqslant 13$ during antiviral therapy, $19 \%$ developed a major depressive episode according to DSM-IV criteria $(n=17,95 \%$ CI $12-28 \%)$ and $12 \%$ had severe depressive syndromes (MADRS score $\geqslant 25$ ) $(n=11,95 \%$ CI $7-21 \%)$ (Table 1).

\section{Baseline factors and INF-associated depression}

Baseline factors were compared between patients with any kind of depressive syndrome during antiviral therapy with PEG-IFN- $\alpha 2 \mathrm{a}$ and ribavirin (MADRS score $\geqslant 13$ ), severe depressive syndrome (MADRS score $\geqslant 25$ ), major depressive episode (defined by DSM-IV criteria) and patients without depression. No differences between patients with depression and those without were found for age, genotype and BMI. However, more women $(n=28$, $65 \%)$ than males $(n=21,44 \%)$ developed depressive syndromes $\left(\right.$ MADRS $\geqslant 13, \chi^{2}=4.166$, d.f. $\left.=1, P 0.041\right)$.

Patients with clinically relevant depression during IFN treatment had significant higher MADRS $(t=-3.858$, d.f. $=89$, $P<0.001)$ and BDI scores $(t=-2.012$, d.f. $=88, P=0.05)$ at baseline and exhibited significantly higher MADRS scores after 2 $(t=-4.155$, d.f. $=89, P<0.001)$ and 4 weeks $(t=-4.861$, d.f. $=88, P<0.001)$ of antiviral treatment. Patients who developed a major depressive episode during antiviral therapy demonstrated higher MADRS scores at baseline $(t=-2.437$, d.f. $=88, P=0.017)$, and after $2(t=-4.245$, d.f. $=89, P<0.001)$ and 4 weeks $(t=-3.071$, d.f. $=89, P=0.013)$ of antiviral treatment. In contrast to this, no significant differences were found between individuals with depression and those without for the BDI score $(P=0.19)$. Patients who developed severe depressive syndromes (MADRS score $\geqslant 25)$ also showed significantly higher MADRS scores at baseline $(t=-2.440$, d.f. $=89, P=0.017)$, as well as after 2 $(t=4.552$, d.f. $=89, P<0.001)$ and 4 weeks $(t=2.976$, d.f. $=80$, $P=0.004)$ of antiviral treatment. No influence on the development of major depressive episodes or a severe depressive syndrome was found for gender, age, genotype and BMI.

Table 2 shows the single-item analysis of the MADRS scale before antiviral treatment was initiated. Patients who developed any kind of an IFN-associated depressive syndrome (MADRS score $\geqslant 13$ ) had significantly higher scores in the following single MADRS items at baseline: apparent sadness $(P=0.009)$, reported sadness $(P=0.002)$, inability to feel $(P=0.015)$, loss of concentration $(P=0.001)$, pessimistic thoughts $(P=0.005)$, reduced sleep $(P=0.050)$, reduced appetite $(P=0.009)$ and lassitude $(P=0.004)$. Patients with major depressive episodes had higher scores on the following five MADRS items at baseline: reduced appetite $(P=0.016)$, loss of concentration $(P<0.001)$, lassitude $(P=0.016)$, pessimistic thoughts $(P=0.001)$ and suicidal thoughts $(P=0.022)$. The development of severe depression (MADRS score $\geqslant 25$ ) during antiviral therapy was associated with higher scores for reduced appetite $(P=0.001)$, loss of concentration $(P=0.001)$, lassitude $(P=0.045)$ and pessimistic thoughts $(t=2.636$, d.f. $=90, P=0.010)$.

We also examined the possible role of cognitive function for the development of depression with the following neuropsychological tests: TMT A, TMT B and the Regensburger word fluency test. Pre-treatment results were compared between patients with and without IFN-associated depressive syndromes. Patients with any clinically relevant depressive syndrome during therapy (MADRS $\geqslant 13)$ showed lower scores on TMT B $(t=2.580$, d.f. $=83$, $P=0.012)$ and semantic word fluency $(t=2.219$, d.f. $=85$,

Table 1 Comparison of pre-treatment demographic and clinical characteristics between patients without and with depressive syndromes during antiviral treatment

\begin{tabular}{|c|c|c|c|c|c|}
\hline Baseline & $\begin{array}{c}\text { All } \\
(n=91)\end{array}$ & $\begin{array}{l}\text { No depression } \\
\quad(n=42)\end{array}$ & $\begin{array}{l}\text { Major depressive } \\
\text { episode }(n=17)\end{array}$ & $\begin{array}{l}\text { MADRS score } \geqslant 13 \\
\quad(n=49)\end{array}$ & $\begin{array}{c}\text { MADRS score } \geqslant 25 \\
(n=11)\end{array}$ \\
\hline Male gender, $n(\%)$ & $48(53)$ & $27(64)$ & $7(41)$ & $21(43)$ & $4(36)$ \\
\hline \multicolumn{6}{|l|}{ Age, years } \\
\hline Mean (s.d.) range & 48.5 (11.3) 21-71 & 47.9 (11.3) 21-66 & 50.8 (9.7) 31-71 & 49 (11.5) 23-71 & 50 (11.0) 31-71 \\
\hline$\leqslant 40$ years, $n(\%)$ & $22(24)$ & $11(26)$ & $1(6)$ & $11(22)$ & $10(91)$ \\
\hline Ethnicity, White, n (\%) & $80(88)$ & $35(83)$ & $16(94)$ & $45(92)$ & $11(100)$ \\
\hline \multicolumn{6}{|l|}{ Body mass index, $\mathrm{kg} / \mathrm{m}^{2}$} \\
\hline Mean (s.d.) range & 25.8 (8) 17-39 & $25.8(4.2) 17-34$ & 24.7 (3.4) 22-36 & 25.8 (10.1) 17-39 & $25.4(4.0) 22-36$ \\
\hline$>25 \mathrm{~kg} / \mathrm{m}^{2}, n(\%)$ & $40(44)$ & $23(55)$ & $5(30)$ & $17(35)$ & $5(46)$ \\
\hline \multicolumn{6}{|l|}{ Genotype, $^{a} n(\%)$} \\
\hline 1 & $59(65)$ & $24(57)$ & $12(71)$ & $35(71)$ & $9(82)$ \\
\hline 2 & $5(5)$ & $3(7)$ & $2(12)$ & $2(4)$ & $1(9)$ \\
\hline 3 & $21(23)$ & $11(26)$ & $2(12)$ & $10(20)$ & $1(9)$ \\
\hline 4 & $6(7)$ & $4(10)$ & $1(6)$ & $2(4)$ & $0(0)$ \\
\hline MADRS, mean (s.d.) range & $2.7(3.9) 0-16$ & $1.4(2.4) 0-10$ & $5.4(5.8) 0-16$ & $4.6(4.8) 0-16$ & $5.9(5.9) 0-16$ \\
\hline Beck Depression Inventory, mean (s.d.) range & $4.0(4.1) 0-18$ & $4.0(3.8) 0-13$ & $6(4.3) 0-15$ & $5.6(4.2) 0-18$ & $5.5(3.7) 0-13$ \\
\hline \multicolumn{6}{|l|}{ Trail Making Test, s: mean (s.d.) range } \\
\hline Test $A$ & 39.5 (16.4) 15-100 & $36.4(11.6)$ 19-65 & 48.2 (19.6) 20-88 & $42.1(19.3) 15-100$ & 59.5 (14.4) 35-88 \\
\hline Test B & 94.6 (36.1) 30-205 & 81.8 (27.2) 35-145 & 109.5 (35.0) 60-190 & 101.8 (40.7) 30-205 & 130.9 (28.1) 100-190 \\
\hline Phonematic word fluency test, mean (s.d.) range & $32.4(10.4) 12-61$ & $29.8(9.3) 13-47$ & 35.6 (11.9) 20-61 & 34.7 (10.9) 12-61 & $37.2(12.5)$ 20-61 \\
\hline Semantic word fluency test, mean (s.d.) range & $22.1(9.4) 7-77$ & $21.5(6.6) 7-35$ & $24.4(15.8) 11-77$ & $22.9(4.9) 18-36$ & $22.4(8.6) 11-34$ \\
\hline $\begin{array}{l}\text { Sandoz Clinical Assessment-Geriatric, } \\
\text { mean (s.d.) range }\end{array}$ & $20.6(4.2) 18-38$ & $19.2(2.6) 18-31$ & $22.6(5.6) 18-33$ & $21.7(4.9) 18-38$ & $22.6(5.6) 18-33$ \\
\hline \multicolumn{6}{|c|}{$\begin{array}{l}\text { MADRS, Montgomery-Åsserg Depression Rating Scale. } \\
\text { a. Hepatitis C virus (HCV subtypes/genotypes } 1,2,3 \text { or } 4 \text { that are important for different treatment strategies: patients with HCV genotype } 1 \text { or } 4 \text { received treatment for } 48 \text { weeks } \\
\text { with pegylated (PEG)-interferon-alpha (IFN- } 2 \text { ) } 2 \mathrm{a}, 180 \mu \mathrm{g} \text { weekly and ribavirin } 1000 \mathrm{mg} \text { daily (body weight }<75 \mathrm{~kg} \text { ) or } 1200 \mathrm{mg} \text { daily (body weight } \geqslant 75 \mathrm{~kg} \text { ). Patients with genotype } 2 \text { or } 3 \\
\text { received PEG-IFN- } \alpha 2 a, 180 \mu \mathrm{g} \text { weekly and ribavirin } 800 \mathrm{mg} \text { daily, for } 24 \text { weeks. }\end{array}$} \\
\hline
\end{tabular}




\begin{tabular}{|c|c|c|c|c|c|}
\hline \multirow[b]{2}{*}{$\begin{array}{l}\text { MADRS single items } \\
\text { at baseline }\end{array}$} & \multicolumn{5}{|c|}{ Mean (s.d.) } \\
\hline & $\begin{array}{c}\text { All } \\
(n=91)\end{array}$ & $\begin{array}{l}\text { No depression } \\
\quad(n=42)\end{array}$ & $\begin{array}{l}\text { Major depression } \\
\text { (DSM-IV) }(n=17)\end{array}$ & $\begin{array}{l}\text { Depression, MADRS } \\
\text { score } \geqslant 13(n=49)\end{array}$ & $\begin{array}{l}\text { Severe depression, MADRS } \\
\text { score } \geqslant 25(n=11)\end{array}$ \\
\hline Apparent sadness & $0.3(0.6)$ & $0.1(0.4)$ & $0.5(0.7)$ & $0.5(0.8)^{\star \star}$ & $0.4(0.7)$ \\
\hline Reported sadness & $0.3(0.7)$ & $0.1(0.3)$ & $0.5(0.9)$ & $0.5(0.8)^{* *}$ & $0.4(0.7)$ \\
\hline Inner tension & $0.4(0.8)$ & $0.3(0.7)$ & $0.5(1.0)$ & $0.5(0.9)$ & $0.5(0.9)$ \\
\hline Reduced sleep & $0.7(1.0)$ & $0.5(0.9)$ & $0.8(1.0)$ & $0.9(1.1)^{*}$ & $1.1(1.1)$ \\
\hline Reduced appetite & $0.3(0.7)$ & $0.1(0.3)$ & $0.6(1.1)^{*}$ & $0.4(0.9)^{\star *}$ & $0.9(1.3)^{\star *}$ \\
\hline Loss of concentration & $0.3(0.7)$ & $0.1(0.5)$ & $0.8(0.9)^{\star *}$ & $0.5(0.7)^{\star *}$ & $0.9(0.9)^{\star *}$ \\
\hline Lassitude & $0.3(0.6)$ & $0.1(0.4)$ & $0.6(0.7)^{*}$ & $0.5(0.7)^{\star *}$ & $0.6(0.7)^{\star}$ \\
\hline Inability to feel & $0.1(0.4)$ & $0.0(0.2)$ & $0.2(0.6)$ & $0.2(0.5)^{\star *}$ & $0.3(0.6)$ \\
\hline Pessimistic thoughts & $0.3(0.7)$ & $0.1(0.4)$ & $0.8(1.1)^{\star \star}$ & $0.5(0.8)^{*}$ & $0.8(1.2)^{\star *}$ \\
\hline Suicidal thoughts & $0.0(0.2)$ & $0.0(0.0)$ & $0.1(0.3)^{*}$ & $0.06(0.2)$ & $0.09(0.3)$ \\
\hline
\end{tabular}

$P=0.029)$ before IFN treatment was started. Patients with a major depressive episode during IFN therapy demonstrated significantly lower scores on TMT A $(t=2.104$, d.f. $=83, P=0.038)$, and semantic word fluency $(t=2.265$, d.f. $=85, P=0.026)$ compared with patients without depression at baseline. Finally, patients with a severe depressive syndrome (MADRS score $\geqslant 25$ ) had significantly lower scores in the TMT A $(t=3.927$, d.f. $=87, P<0.001)$ and TMT B at baseline $(t=3.105$, d.f. $=83, P=0.003)$.

With respect to the SCAG, patients with IFN-associated depressive syndrome and major depressive episodes had significant higher scores before INF therapy was started (MADRS $\geqslant 13$ : $t=2.901$, d.f. $=88, P=0.005$; major depressive episodes: $t=2.256$, d.f. $=88, P=0.027)$.

\section{Prediction of INF-associated depression}

A multivariable regression analysis was performed to identify those risk factors that maximally discriminated between patients who developed any kind of a clinically relevant depressive syndrome (MADRS scores $\geqslant 13$ ) during IFN treatment and those who remained without clinically relevant depressive mood changes (Fig. 1). A baseline MADRS score of 3 or higher was associated with a 6.4-fold increased higher risk for the development of an IFN-associated clinically relevant depressive syndrome (95\% CI 1.7-24.0, $P=0.006$ ). A BMI less than 25 at baseline resulted in a significantly higher risk for the development of a clinically relevant depressive syndrome $(\mathrm{OR}=4.63,95 \% \mathrm{CI}$ $1.5-14.8, P=0.010)$. In contrast, logistic regression analysis revealed that neither major depressive episodes according to DSM-IV criteria nor severe depressive syndromes (MADRS score $\geqslant 25$ ) were associated with any baseline factors (data not shown).

A multiple logistic regression analysis was used again to calculate odds ratios of the incidence of INF-associated depressive syndromes (MADRS $\geqslant 13$, major depressive episodes, MADRS $\geqslant 25$ ) adjusted for TMT A, TMT B, Regensburger verbal fluency test semantic and phonematic word fluency and the SCAG score. Reduced performance on the TMT A (OR $=1.1,95 \%$ CI 1.0-1.1, $P=0.027)$ and TMT B $(\mathrm{OR}=1.0,95 \%$ CI $1.0-1.1, P=0.033)$ predicted the development of severe depression (MADRS $\geqslant 25$ ) during antiviral treatment. Higher scores on the SCAG $(\mathrm{OR}=1.23,95 \%$ CI $1.0-1.4, P=0.014)$ was found to be the only
Odds ratio $(95 \% \mathrm{Cl})$

Factor

Gender: female (v. male)

Age: $>50$ years $(v . \leqslant 50$ years)

BMl: $<25(v . \geqslant 25)$

Genotype: 1/4 (v. 2/3)

MADRS: $\geqslant 3($ v. $<3)$

BDI: $\geqslant 4(v .<4)$

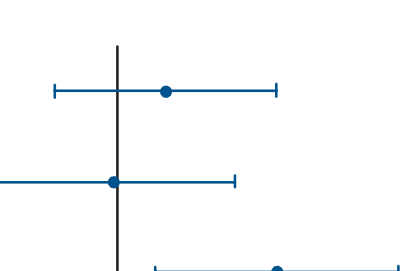

$0.96(0.30-3.05)$

$4.64(1.45-14.80)$

$3.48(0.87-13.89)$

$6.43(1.72-23.97)$

$1.67(0.53-5.29)$
Common odds ratio test

0.010

0.006

0.385

Fig. 1 Odds ratios for developing any clinically relevant depressive syndrome (Montgomery-Åsberg Depression Rating Scale (MADRS) scores $\geqslant 13$ ) during interferon treatment.

BMI, body mass index; BDI, Beck Depression Inventory. 
relevant risk factor for the development of an INF-associated depressive syndrome (MADRS $\geqslant 13$ ) during antiviral therapy.

\section{Decision tree for the prediction of INF-associated depression}

As a MADRS score of 3 or higher was determined as the only significant predictor for an INF-associated depressive syndrome, we analysed the individual items of the MADRS. Items were included in the decision tree regression analyses in order to define cut-off points for each symptom of depression on the MADRS scale at baseline to discriminate between patients with or without an IFN-induced depressive syndrome (Fig. 2). Patients were classified according to their values on the MADRS items as independent variables, and according to the emergence of a depressive syndrome (MADRS $\geqslant 13$ ). The final decision tree analysis distinguished between patients with clinical depression and those without with a sensitivity of $73.6 \%$. For developing INF-induced depression (MADRS $\geqslant 13$ ), statistically significant predictors at baseline evaluation were reported sadness, loss of concentration, pessimistic thoughts and reduced sleep.

In total, $89 \%(n=16)$ of patients with a baseline score for 'reported sadness' greater than 0 developed a clinically relevant depressive syndrome during IFN-therapy. Further analyses revealed that $75 \%(n=9)$ of patients without reported sadness, but with loss in concentration (score $>0$ ) developed an INF-associated depressive syndrome (MADRS $\geqslant 13$ ). The prediction rate for absence of depression with INF-treatment (specificity) for this decision tree was $73.8 \%$.

\section{Discussion}

\section{Main findings}

Clinically relevant depressive symptoms emerged in up to $70 \%$ of patients with chronic hepatitis $\mathrm{C}$ who received antiviral therapy including IFN- $\alpha^{4,17}$ Two recently published meta-analyses demonstrated that pre-emptive antidepressant treatment with SSRIs significantly reduces the incidence of IFN-associated depression. ${ }^{5,18}$ However, strategies are demanded that help to discriminate between patients at risk of INF-induced depression and those who are not. Our results show significant differences at baseline between patients with and without IFN-associated depressive syndromes. Pre-existing subclinical depressive mood (MADRS $\geqslant 3$ ) resulted in a 6.4 -fold increased risk of clinically relevant IFN-associated depressive syndromes in patients without

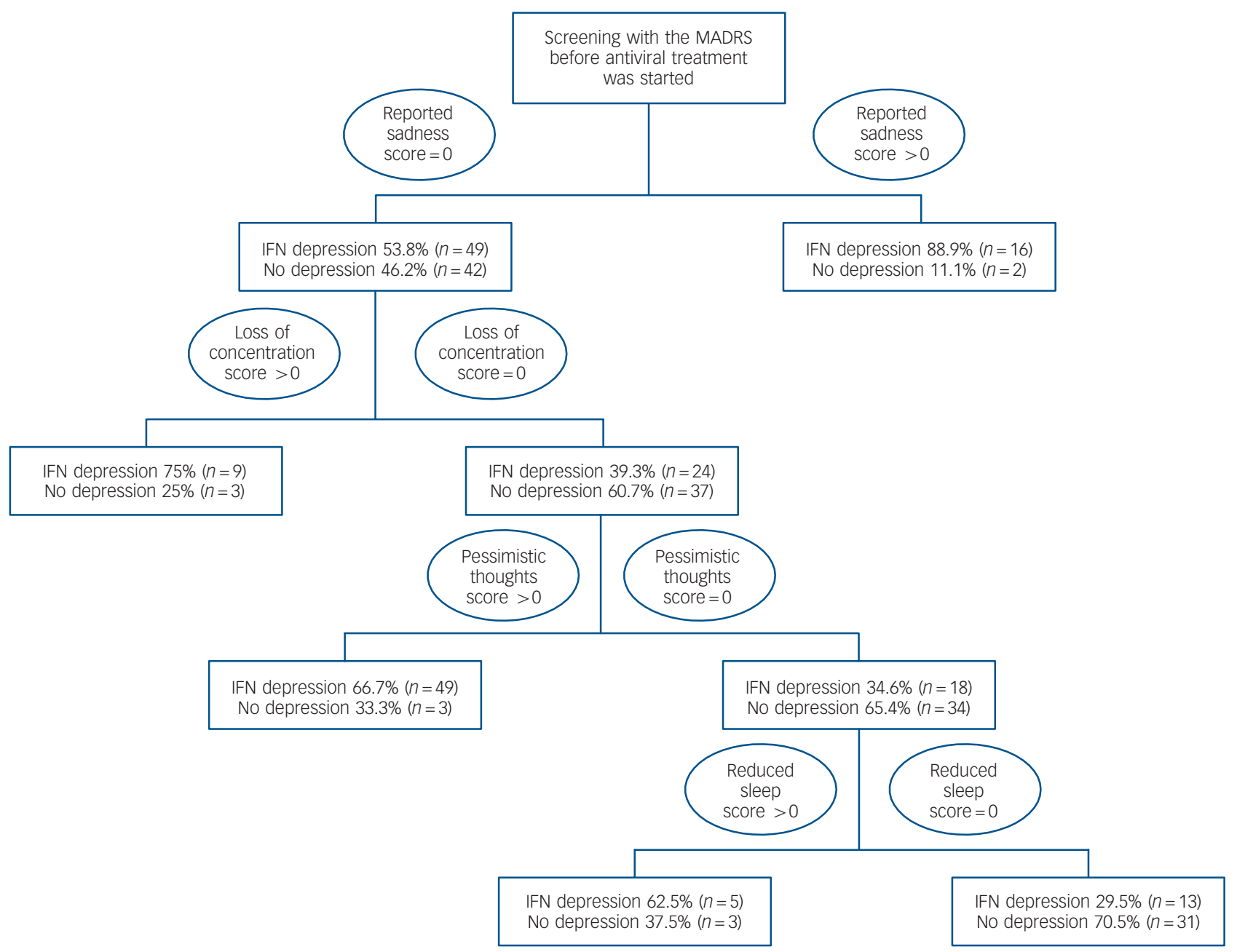

Fig. 2 Decision tree analyses using single symptom items on the Montgomery-Åsberg Depression Rating Scale (MADRS) to predict interferon-alpha (INF- $\alpha$ )-induced depression during antiviral treatment.

Four MADRS items were found by decision tree analysis to predict IFN- $\alpha$ associated depression: reported sadness, loss of concentration, pessimistic thoughts and reduced sleep, with the highest predictive value for the item reported sadness. The final decision tree analysis distinguished between patients with clinical depression from those without, with a sensitivity of $73.6 \%$ (correct predicted depression rate $73.5 \%$, correct predicted non-depression rate $73.8 \%$ ). The overall IFN depression rate was $53.8 \%$ ( $n=49$ ) compared with $46.2 \%(n=42)$ for those without interferon-induced depression. IFN- $\alpha$ depression, INF- $\alpha$-induced depression defined by MADRS $\geqslant 13$. 
a history of psychiatric disorders. By using decision tree analysis, four single items of the MADRS scale - reported sadness, loss of concentration, pessimistic thoughts and reduced sleep - stood out in predicting the development of a clinically relevant depressive syndrome (MADRS $\geqslant 13$ ) with a sensitivity of $73.8 \%$. A total of $89 \%(n=16)$ of patients with reported sadness at baseline (MADRS single item, score $>0$ ) developed a clinically relevant depressive syndrome during IFN-therapy.

Our results are in line with previous studies demonstrating that patients with higher MADRS scores at baseline have a higher risk for the development of depressive symptoms during cytokine therapy. ${ }^{3,19-21}$ Capuron and colleagues identified similar mood (sadness, pessimistic thoughts) and behavioural symptoms (sleep disturbance) as significant predictors of depressive symptoms at the end of the first month of cytokine treatment. ${ }^{20}$ These findings support the notion that even subclinical deterioration of the affective state of patients before the initiation of INF therapy may indicate an increased individual vulnerability for the development of clinically significant IFN-associated depressive symptoms. Interestingly, the number or severity of these symptoms seem not to be decisive factors. Rather, our results indicate an 'all-or-nothing' effect: the risk of developing clinically relevant depressive symptoms during IFN treatment was increased whenever patients reported any kind of subclinical depressive symptoms, especially sadness and in addition sleep disturbance, pessimistic thoughts and lack of concentration. Thus, screening of patients for any degree of these pre-existing symptoms is advisable.

The predictive value of subclinical depressive symptoms before the beginning of interferon therapy shows similarities with the concept of subsyndromal symptomatic depression. This has been defined as minimal depressive symptoms below the diagnostic threshold for minor, dysthymic or major depressive disorders. ${ }^{22-24}$ Subsyndromal symptomatic depression is frequently observed in patients with unipolar major depressive disorder and is considered as an integral component of the symptomatic course of illness. ${ }^{23,25}$ In addition, the presence of subsyndromal symptomatic depression shows significant associations with a higher prevalence of future and past major depressive episodes. ${ }^{24,26-28}$ A similar relationship seems to exist for INF-associated depression and subclinical depressive symptoms before the beginning of antiviral therapy. However, it should be emphasised that despite major depression being a serious and highly prevalent side-effect of INF therapy, most affected patients will respond to acute treatment with SSRIs, ${ }^{29}$ or depression might be prevented by pre-emptive antidepressant treatment. ${ }^{5,6}$ Thus, even with the increased risk for depression, it is not indicated to exclude patients with minimal depressive symptomatology from antiviral treatment with IFN $-\alpha .{ }^{3}$

Another important and novel finding is that severe depression can be predicted by neuropsychological tests measuring cognitive flexibility (TMT A and B). It was evident that inferior cognitive performance predicts especially severe depression before the start of INF treatment. This increased vulnerability to severe depression was even found in patients without a history of any affective disorders. With respect to subjective cognitive problems as measured by the SCAG, patients with IFN-associated depressive syndromes $($ MADRS $\geqslant 13$ ) had significantly higher scores before INF treatment was started.

\section{Cognitive impairment and HCV infection}

Cognitive difficulties are associated with impaired work productivity. ${ }^{30}$ Of note, treatment-naive patients with chronic hepatitis $\mathrm{C}$ infection have an already increased risk of mild cognitive impairment. ${ }^{3,31}$ Chronic infection with $\mathrm{HCV}$ is suspected to cause lasting changes to the immune system and to neurotransmission, leading to mild cognitive impairment. ${ }^{4,32}$ Different hypotheses have been proposed to explain the occurrence of cognitive impairment in HCV infection, such as a direct action of the virus on the central nervous system (CNS), a delayed action of the virus on the CNS mediated by viral replication in neurons and cognitive deterioration as a side-effect of the inflammatory process. ${ }^{33}$ In contrast, a recently published study demonstrated no evidence of an association between HCV infection and cognitive impairment. ${ }^{34}$ The authors argue that a lack of rigorous selection criteria in previous studies may have resulted in an overestimation of the prevalence of cognitive impairment associated with HCV by including patients with a history of alcohol and/or illegal drug misuse, patients using psychotropic drugs or patients with depression. ${ }^{34}$ However, our trial, which excluded patients with psychiatric disorders, now confirms the presence of at least mild cognitive impairment in a subgroup of treatment-naive patients with chronic hepatitis $\mathrm{C}$ independent from depressive symptoms.

On the other hand, and independent from HCV infection, two recent meta-analyses support the presence of cognitive impairment in patients with major depressive disorder. ${ }^{35,36}$ These studies demonstrate that executive function mediated by the prefrontral cortex and measured with the TMT B is affected by depression, which may have an impact on cognitive performance. The finding that a reduced performance on TMT A and TMT B predicts the development of severe depression (MADRS $\geqslant 25$ ) lends further support to a depression-executive dysfunction syndrome. ${ }^{37}$ Executive dysfunction is also one of the clinical expressions of abnormalities in the frontostriatal-limbic circuitry that might predispose to late-life depression.

\section{BMI}

In our trial a $\mathrm{BMI} \geqslant 25$ at baseline resulted in a significantly reduced risk of developing clinically relevant depression with INF treatment. Obesity is one of the core characteristics that has been associated with lower sustained viral response rates in patients with chronic hepatitis ${ }^{38}$ and might be associated with a higher relapse rate. ${ }^{39}$ Therapy with IFN- $\alpha$ in these patients might have less clinical antiviral effects, resulting in lower rates of neuropsychiatric side-effects.

\section{Limitations}

Although the present study generally supports the utility of the TMT as a screening tool for HCV patients before the beginning of IFN therapy, further investigations are clearly required. For example, additional cognitive tests should be conducted in future studies to evaluate whether other cognitive functions also predict severe IFN-associated depression (for example, attention or memory). A more extensive evaluation would provide a stronger and more rigorous basis for assessing the potential impact of cognitive functions at the beginning of INF therapy in relation to INF-associated depression. Although a reasonable number of patients was included, numbers did not allow for a more precise identification of risk factors for developing DSM-IV major depressive episodes or severe depression (MADRS $\geqslant 25$ ). Finally, multiple testing might limit the results.

\section{Implications}

In summary, our data demonstrate that pre-existing subclinical depressive mood significantly increases the risk of clinically relevant IFN-associated depressive syndromes in patients without a history of psychiatric disorders. Mild cognitive disturbances are an independent risk factor for the development of severe depression. Scoring on the MADRS single items reported sadness, 
loss of concentration, pessimistic thoughts and reduced sleep prior to treatment, or a MADRS total score $>3$ at baseline may identify patients at risk of treatment-emergent depression. Pre-emptive treatment with antidepressants is recommended in these high-risk patients.

Susanne Sarkar, PhD, Department of Psychiatry and Psychotherapy, Charité-Universitätsmedizin Berlin, Campus Charité, Mitte, Berlin, and Department and Outpatient Clinic of Medical Psychology, Center of Psychosocial Medicine, University Medical Center Hamburg-Eppendorf, Hamburg; Rahul Sarkar, MD, Department of Psychiatry and Psychotherapy, Asklepios Westklinikum Hamburg-Klinikum, Hamburg; Thomas Berg, MD, Department of Gastroenterology und Rheumatology, Section of Hepatology, Universitätsklinikum, Leipzig; Martin Schaefer, MD, Department of Psychiatry and Psychotherapy, Charité-Universitätsmedizin Berlin, Campus Charité, Mitte, Berlin, and Department of Psychiatry, Psychotherapy and Addiction Medicine, Kliniken Essen-Mitte, Essen, Germany

Correspondence: Martin Schaefer, MD, Department of Psychiatry, Psychotherapy and Addiction Medicine, Kliniken Essen-Mitte, Henricistr. 92, 45136 Essen, Germany. Email: m.schaefer@kliniken-essen-mitte.de

First received 18 Nov 2013, final revision 17 Jun 2014, accepted 19 Jun 2014

\section{Acknowledgements}

We thank Professor Heinz Grunze for a critical review of the paper and helpful advice.

\section{References}

1 Dieperink E, Willenbring M, Ho SB. Neuropsychiatric symptoms associated with hepatitis C and interferon alpha: a review. Am J Psychiatry 2000; 157 867-76.

2 Schaefer M, Engelbrecht MA, Gut O, Fiebich BL, Bauer J, Schmidt F, et al. Interferon alpha (IFNalpha) and psychiatric syndromes: a review. Prog Neuropsychopharmacol Biol Psychiatry 2002; 26: 731-46.

3 Schaefer M, Capuron L, Friebe A, Diez-Quevedo C, Robaeys G, Neri S, et al. Hepatitis $C$ infection, antiviral treatment and mental health: a European expert consensus statement. J Hepatol 2012; 57: 1379-90.

4 Schaefer M, Hinzpeter A, Mohmand A, Janssen G, Pich M, Schwaiger M, et al Hepatitis $C$ treatment in "difficult-to-treat" psychiatric patients with pegylated interferon-alpha and ribavirin: response and psychiatric side effects. Hepatology 2007; 46: 991-8.

5 Sarkar S, Schafer M. Antidepressant pretreatment for the prevention of interferon alfa-associated depression: a systematic review and metaanalysis. Psychosomatics 2014; 55: 221-34.

6 Schaefer M, Sarkar R, Knop V, Effenberger S, Friebe A, Heinze L, et al Escitalopram for the prevention of peginterferon-alpha2a-associated depression in hepatitis $\mathrm{C}$ virus-infected patients without previous psychiatric disease: a randomized trial. Ann Intern Med 2012; 157: 94-103.

7 Gumnick JK, Nemeroff, CB. Problems with currently available antidepressants. J Clin Psychiatry 2000, 61 (suppl 10): 5-15.

8 American Psychiatric Association. Diagnostic and Statistical Manual of Mental Disorder (4th edn) (DSM-IV). APA, 1994.

9 Sheehan DV, Lecrubier $\mathrm{Y}$, Sheehan $\mathrm{KH}$, Amorim $\mathrm{P}$, Janavs J, Weiller $\mathrm{E}$, et al. The Mini-International Neuropsychiatric Interview (M.I.N.I.): the development and validation of a structured diagnostic psychiatric interview for DSM-IV and ICD-10. J Clin Psychiatry 1998; 59 (suppl 20): 22-33.

10 First MB, Spitzer RL, Gibbon M, Williams JBW. Structured Clinical Interview for DSM-IV Axis I Disorders, Clinician Version (SCID-CV). American Psychiatric Press, 1996

11 Montgomery SA, Asberg M. A new depression scale designed to be sensitive to change. Br J Psychiatry 1979; 134: 382-9.

12 Beck AT, Ward $\mathrm{CH}$, Mendelson M, Mock J, Erbaugh J. An inventory for measuring depression. Arch Gen Psychiatry 1961, 4: 561-71.

13 Zimmerman M, Posternak MA, Chelminski I. Implications of using different cut-offs on symptom severity scales to define remission from depression. Int Clin Psychopharmacol 2004; 19: 215-20.

14 Reitan RM, Wolfson D. The Halstead-Reitan Neuropsychological Test Battery Therapy and Clinical Interpretation. Neuropsychological Press, 1985.

15 Aschenbrenner S, Tucha O, Lange KW. Regensburger Wortflüssigkeits-test (RWT). Hogrefe Verlag, 2000.
16 Shader RI, Harmatz JS, Salzman C. A new scale for clinical assessment in geriatric populations: Sandoz Clinical Assessment - Geriatric (SCAG). J Am Geriatr Soc 1974; 22: 107-113

17 Schafer A, Wittchen HU, Seufert J, Kraus MR. Methodological approaches in the assessment of interferon-alfa-induced depression in patients with chronic hepatitis C - a critical review. Int J Methods Psychiatr Res 2007; 16: 186-201.

18 Jiang HY, Deng M, Zhang YH, Chen HZ, Chen Q, Ruan B. Specific serotonin reuptake inhibitors prevent interferon- $\alpha$-induced depression in patients with hepatitis C: a meta-analysis. Clin Gastroenterol Hepatol 2014; 12: 1452-60.

19 Capuron $L$, Ravaud A. Prediciton of the depressive effects of interferon alfa therapy by the patient's initial affective state. N Engl J Med 1999; 340: 1370.

20 Capuron L, Ravaud A, Miller AH, Dantzer R. Baseline mood and psychosocial characteristics of patients developing depressive symptoms during interleukin-2 and/or interferon-alpha cancer therapy. Brain Behav Immun 2004; 18: 205-13.

21 Miyaoka H, Otsubo T, Kamijima K, Ishii M, Onuki M, Mitamura K. Depression from interferon therapy in patients with hepatitis C. Am J Psychiatry 1999; 156: 1120.

22 Judd LL, Rapaport MH, Paulus MP, Brown JL. Subsyndromal symptomatic depression (SSD): a new mood disorder? J Clin Psychiatry 1994; 55: 18-28.

23 Judd LL, Paulus MP, Wells KB, Rapaport MH. Socioeconomic burden of subsyndromal depressive symptoms and major depression in a sample of the general population. Am J Psychiatry 1996; 153: 1411-7.

24 Judd LL, Akiskal HS, Paulus MP. The role and clinical significance of subsyndromal depressive symptoms (SSD) in unipolar major depression. J Affect Disord 1997; 45: 5-17.

25 Judd LL, Akiskal HS, Maser JD, Zeller PJ, Endicott J, Coryell W, et al. A prospective 12-year study of subsyndromal and syndromal depressive symptoms in unipolar major depressive disorders. Arch Gen Psychiatry. 1998; 55: 694-700.

26 Broadhead WE, Blazer DG, George LK, Tse LK. Depression, disability days and days lost from work in a prospective epidemiologic survey. JAMA 1990; 264: 2524-8.

27 Howarth E, Johnson J, Klerman GL, Weissmann MM. Depressive symptoms as relative and attributable risk factors for first-onset major depression. Arch Gen Psychiatry 1992; 49: 817-23.

28 Sherbourne CD, Wells KB, Hays RD, Rogers W, Burnam MA, Judd LL. Subthreshold depression and depressive disorder: clinical characteristics of general medical and mental health specialty outpatients. Am J Psychiatry 1994; 151: 1777-84.

29 Kraus MR, Schafer A, Schoettker K, Keicher C, Weissbruch B, Hofbrauer I, et al. Therapy of interferon-induced depression in chronic hepatitis $C$ with citalopram: a randomised, double-blind, placebo-controlled study. Gut 2008; 57: 531-6.

30 Jaeger J, Berns S, Uzelac S, Davis-Conway S. Neurocognitive deficits and disability in major depressive disorder. Psychiatry Res 2006; 145: 39-48.

31 Tillmann $\mathrm{HL}$, Wiese M, Braun $\mathrm{Y}$, Wiegand J, Tenckhoff S, Mössner J, et al. Quality of life in patients with various liver diseases: patients with HCV show greater mental impairment, while patients with PBC have greater physical impairment. J Viral Hepat 2011; 18: 252-61.

32 Forton DM, Hamilton G, Allsop JM, Grover VP, Wesnes K, O'Sullivan C, et al. Cerebral immune activation in chronic hepatitis $C$ infection: a magnetic resonance spectroscopy study. J Hepatol 2008; 49: 316-22.

33 Senzolo M, Schiff S, D'Aloiso CM, Crivellin C, Cholongitas E, Burra P, et al. Neuropsychological alterations in hepatitis $C$ infection: the role of inflammation. World J Gastroenterol 2011; 17: 3369-74.

34 Abrantes J, Torres DS, de Mello CE. Patients with hepatitis C infection and normal liver function: an evaluation of cognitive function. Postgrad Med J 2013; 89: 433-9.

35 McDermott LM, Ebmeir KP. A meta-analysis of depression severity and cognitive function. J Affect Disord 2009; 119: 1-8.

36 Snyder HR. Major depressive disorder is associated with broad impairments on neuropsychological measures of executive function: a meta-analysis and review. Psychol Bull 2012; 28: 1-52.

37 Alexopoulos GS. Frontostriatal and limbic dysfunction in late-life depression. Am J Geriatr Psychiatry 2002; 10: 687-95.

38 Negro F, Clément S. Impact of obesity, steatosis and insulin resistance on progression and response to therapy of hepatitis C. J Viral Hepat 2009; 16: 681-8.

39 Rivero-Juarez A, Mira JA, Camacho A, Neukam K, Perez-Camacho I, Caruz A, et al. Baseline risk factors for relapse in HIV/HCV co-infected patients treated with PEG-IFN/RBV. Infection 2013; 41: 21-6. 